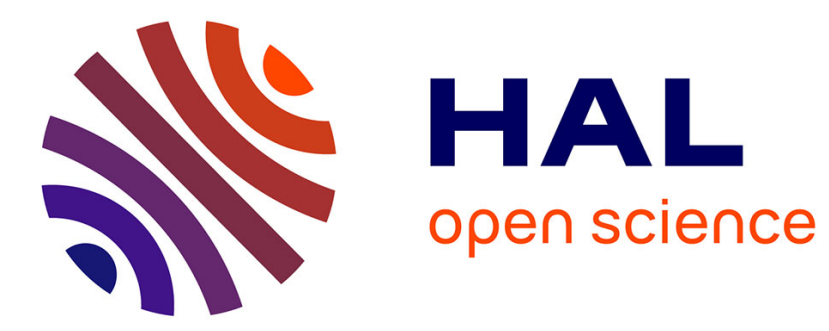

\title{
Integrated Tool-Chain Concept for Automated Micro-optics Assembly
}

Sebastian Haag, Tobias Müller, Christoph Pallasch, Christian Brecher

\section{To cite this version:}

Sebastian Haag, Tobias Müller, Christoph Pallasch, Christian Brecher. Integrated Tool-Chain Concept for Automated Micro-optics Assembly. 7th International Precision Assembly Seminar (IPAS), Feb 2014, Chamonix, France. pp.37-46, 10.1007/978-3-662-45586-9_6 . hal-01260727

\section{HAL Id: hal-01260727}

\section{https://hal.inria.fr/hal-01260727}

Submitted on 22 Jan 2016

HAL is a multi-disciplinary open access archive for the deposit and dissemination of scientific research documents, whether they are published or not. The documents may come from teaching and research institutions in France or abroad, or from public or private research centers.
L'archive ouverte pluridisciplinaire HAL, est destinée au dépôt et à la diffusion de documents scientifiques de niveau recherche, publiés ou non, émanant des établissements d'enseignement et de recherche français ou étrangers, des laboratoires publics ou privés. 


\title{
Integrated Tool-chain Concept for Automated Micro-optics Assembly
}

\author{
Sebastian Haag $^{1 *}$, Tobias Müller ${ }^{1}$, Christoph Pallasch ${ }^{1}$, Christian Brecher $^{1}$ \\ ${ }^{1}$ Fraunhofer Institute for Production Technology IPT, Steinbachstr. 17, 52074 Aachen, Germany \\ * corresponding author: sebastian.haag@,ipt.fraunhofer.de
}

\begin{abstract}
The work presented in this paper aims for a significant reduction of process development and production ramp up times for the automated assembly of micro-optical modules and systems. The approach proposed bridges the gap between the product development phase and the realization of automation control through integration of established software tools such as optics simulation and CAD modeling as well as through introduction of novel software tools and methods to efficiently describe active alignment strategies. The focus of the paper is put on the formalized specification of product configurations as a basis for the engineering of automated assembly processes. The concepts are applied to industrial use-cases. The paper concludes with an overview of the application of the concepts in an engineering tool-chain as well as an outlook on the next development steps.
\end{abstract}

\section{Introduction}

Photonics technologies are key technologies of the $21^{\text {st }}$ century enabling innovative applications in demanding and societal relevant domains. In many cases, photonic applications belong to the field of high-technology applications with small and medium production volumes. In order to respond to increasing competitive pressure in production of micro-optical systems and lasers, flexible, autonomous, and efficient solutions for assembly systems have to be developed [1]. Competitive small and medium size volume production can only be achieved based on an increased degree of automation as automation can reduce costs while realizing highly reliable processes and high product quality as well as improved working conditions. However, a high degree of automation still correlates negatively with the flexibility of a production system which can lead to a major negative impact on the return on investment calculation. Recently, promising progress has been made developing modular and reconfigurable assembly systems [9, 11]. There is a significant lack of corresponding software solutions to allow for an efficient exploitation of the available flexibility as promised by computer-aided process planning (CAPP).

In order to fulfil precision requirements and due to manufacturing tolerances and finite accuracy of the positioning systems, the assembly of micro-optical systems requires the use of sensor-guided processes such as vision-based component pickup, passive alignment exploiting geometrical features through image-processing, and active alignment optimizing the optical function towards a desired value. The use of sensors requires the processing and analysis of signals and in some cases reasoning in order to extract information from it which drastically increases the complexity of process planning and commissioning. Thus, the required engineering time is high and nullifies the benefit of automation. This is the motivation for ongoing research activities which intends to develop and validate an integrated development environment (IDE) for automated precision assembly processes bridging the gaps between computer-aided product development, virtual process planning and assembly execution by means of methods and software tools for implementing complex sensor-based handling and alignment processes.

The assembly of micro-optical systems is still dominated by manual operations. Feasibility of automation has been proven for many cases such as resonator mirror alignment, focus optics alignment, fast-axis collimator alignment for diode laser bars as well as for multi-single emitter diode laser modules $([2,3,4,5,6])$. Investment and implementation costs are still high and mean a high risk - especially for small and medium sized companies. An engineering gap regarding the information transfer from product development to the subsequent engineering phases of process development and assembly system configuration and commissioning can be stated. This gap needs to be overcome in order to allow automation technology to achieve a breakthrough in the production of optical systems such as lasers. The work presented in this paper strives for closing the identified engineering gap by proposing a standardized and yet flexible approach for engineering such assembly solutions supported by corresponding software tools which do not exist in the domain of laser system production. The main task identified by the authors is the introduction of a flexible engineering methodology for planning and commissioning the automated assembly of optical systems and therefore the integration of existing tools and the conceptual design and the prototypal implementation of novel tools for bridging existing engineering gaps.

\section{Concept of Hybrid Assembly Process Planning for Optical Systems}

This paper proposes a hybrid approach for assembly process planning for optical systems in which parts of the engineering can be automated as in CAPP and other engineering tasks are implemented or optimized by 
operators. ElMaraghy defines in [11] the term of micro-process planning as the specification of detailed parameters for individual operations. This definition corresponds with the intent of the proposed approach which integrates well-established engineering tools (for CAD modeling, optical simulation, PLC and robot programming) as well as novel software modules for the specification of sensor-guided process parameters through standardized communication interfaces and data formats. An iterative approach of virtual and physical engineering steps is proposed in order to provide sufficient flexibility during the process planning phase. Virtual engineering steps comprise offline and computer-aided development efforts. Physical engineering steps stand for development efforts carried out physically, e.g. the refinement of an active alignment process using real laser and camera (instead of simulation). The term 'online' is avoided because the authors use that term for activities in the actual production system.

\begin{tabular}{|c|c|c|c|c|c|}
\hline $\begin{array}{c}\text { Product Design } \\
\text { (virtual) } \\
\text { PD }\end{array}$ & $\begin{array}{l}\text { Product } \\
\text { Prototyping } \\
\text { (physical) } \\
\text { PP }\end{array}$ & $\begin{array}{l}\text { Virtual Platform- } \\
\text { independent } \\
\text { Process Planning } \\
\text { vPIPP }\end{array}$ & $\begin{array}{l}\text { Physical Platform- } \\
\text { independent } \\
\text { Process Planning } \\
\text { pPIPP }\end{array}$ & $\begin{array}{c}\text { Virtual Platform- } \\
\text { specific Process } \\
\text { Commissioning } \\
\text { VPSPC }\end{array}$ & $\begin{array}{c}\text { Physical Platform- } \\
\text { specific Process } \\
\text { Commissioning } \\
\text { pPSPC }\end{array}$ \\
\hline $\begin{array}{l}\text { - Design of optical } \\
\text { system } \\
\text { - CAD including } \\
\text { thermal analysis }\end{array}$ & $\begin{array}{l}\text { - Assembly and } \\
\text { evaluation of } \\
\text { prototypes } \\
\text { - Design review }\end{array}$ & $\begin{array}{l}\text { - T2B KC-specification } \\
\text { - Assembly sequence } \\
\text { generation } \\
\text { - Alignment-in-the- } \\
\text { loop }\end{array}$ & $\begin{array}{l}\text { - Realization of } \\
\text { alignment strategy } \\
\text { - Enhancement of } \\
\text { alignment towards } \\
\text { robustness }\end{array}$ & $\begin{array}{l}\text { - Teaching in VR } \\
\text { - Allocation of } \\
\text { represented } \\
\text { hardware entities }\end{array}$ & $\begin{array}{l}\text { - Integration of hard- } \\
\text { and software }\end{array}$ \\
\hline
\end{tabular}

Fig. 1: Overview of engineering elements for hybrid planning and commissioning the automated assembly of optical systems

Fig. 1 provides an overview of the elements of the proposed engineering platform. Product design (PD) is usually carried out using computer-aided (virtual) methods. Early physical realization of the product as a prototype allows evaluation of the design and possibly design improvements. For process planning and commissioning the authors propose a two-stage approach analogue to model-driven engineering approaches for maximizing re-usability [12]. The first stage is a platform-independent view (xPIxx) on the task while the second stage is platform-specific (xPSxx). Furthermore, process planning and commissioning are both proposed to be subdivided in a virtual as well as a physical branch. This framework leads to four engineering elements:

- Virtual platform-independent process planning (vPIPP): This engineering step carries out top-to-bottom (T2B) specification of key characteristics in a formal way and uses that information for deriving an assembly sequence. For the development and verification of specific assembly steps, tools such as the proposed alignment-in-the-loop can be used to virtually specify and verify complex tasks offline.

- $\quad$ Physical platform-independent process planning (pPIPP): On a dedicated prototyping platform individual process steps such as active alignment pre-planned in the virtual environment can be executed and validated. Discrepancies between ideal models and the real world can be identified and compensated this way. A possible solution to overcome such discrepancies is the integration of visible reference marks for local referencing between model and hardware.

- Virtual platform-specific process commissioning (vPSPC): For mapping the assembly task specification consisting of an assembly sequence and virtually planned process steps to an assembly platform, the concept proposes the use of Virtual Production Systems (VPS). VPS's can be used for teaching coordinates in the model environment. High-precision tasks require the use of sensor-guidance based on visible reference marks.

- $\quad$ Physical platform-specific process commissioning (pPSPC): The final step of commissioning takes place on the production platform in a drastically reduced way complexity and ramp up time.

Fig. 2 depicts the engineering phases of product development, process planning, and commissioning of the assembly task in a slightly different way than in the previous section. Here, the paths of information exchange are illustrated by arrows. For an efficient engineering process, it must be ensured that all phases have welldefined interfaces for passing on and exchanging information between each other. Note, that no direct links for information exchange are depicted between the physical engineering steps (PP, pPIPP and pPSPC). Information must be fed back to the virtual environment in the models in order to refine the model-driven approach. 


\begin{tabular}{|c|c|c|}
\hline $\begin{array}{l}\text { Product } \\
\text { Development }\end{array}$ & $\begin{array}{l}\text { Functional product design } \\
\text { (optical modelling) } \\
\text { CAD modelling }\end{array}$ & $\begin{array}{l}\text { Prototype assembly } \\
\text { Design review }\end{array}$ \\
\hline $\begin{array}{l}\text { Process } \\
\text { Planning }\end{array}$ & $\begin{array}{l}\text { Specification of key characteristics VPIPP } \\
\text { Synthesis of assembly sequence } \\
\text { Alignment-in-the-loop }\end{array}$ & $\begin{array}{l}\text { Validation and refinement of } \quad p P I P P \\
\text { alignment and sensor-based process steps }\end{array}$ \\
\hline $\begin{array}{l}\text { Commission- } \\
\text { ing }\end{array}$ & $\begin{array}{l}\text { Allocation of resources } \\
\text { Virtual teaching }\end{array}$ & $\begin{array}{l}\text { System integration } \quad p P S P C \\
\text { Commissioning on production system }\end{array}$ \\
\hline
\end{tabular}

Fig. 2: Engineering phases for the assembly of micro-optical systems. Arrows indicate flow of information.

\subsection{Overview of the integrated tool-chain}

For the development of the photonic products, established software tools are available such as optical simulation tools and CAD modeling tools (Fig. 3, left column). Assembly process planning for micro-optical systems comprises the sequence of assembly steps and the planning of processes such as part feeding and alignment. The product configurator edits formalized representation of the liaison diagram and therefore bridges the gap between product development and process planning (Fig. 3, middle column). In the VPS resources are assigned and the assembly sequence is verified regarding logic and collisions (Fig. 3, right column).

\begin{tabular}{|c|c|c|}
\hline PD & VPIPP & VPSPC \\
\hline $\begin{array}{c}\text { Optical modeling and } \\
\text { simulation }\end{array}$ & $\begin{array}{c}\text { Product configurator } \\
\text { (specification of KC's) }\end{array}$ & $\begin{array}{c}\text { Virtual Production } \\
\text { System }\end{array}$ \\
\hline $\begin{array}{c}\text { Mechanical design and } \\
\text { thermal analysis }\end{array}$ & Alignment-in-the- \\
\hline & $\begin{array}{c}\text { loop } \\
\text { Toolkit for detection } \\
\text { of reference marks }\end{array}$ \\
\hline
\end{tabular}

Fig. 3: Overview of tools in IDE

\subsection{Formalization of the product configuration}

Product development consists of several phases. In a first step, functional and non-functional requirements are defined from a customer point of view. In a second step, the defined requirements are transformed into technical requirements specifications. In a third step, for optical systems, an optical model is created and assembly tolerances are analyzed through simulation. As a result of this step the optical elements are chosen and the product can be designed in CAD regarding its outer and inner dimensions. Furthermore, the mechanical design of laser systems is optimized regarding its thermal behavior by means of thermal analysis. Professional and wellestablished software is available for all of these steps.

In the case of optical systems, special attention needs to be paid to the high precision demands often reaching in the sub-micrometer domain. Process steps such as handling, alignment and bonding of parts in many cases need sensor-based strategies compensating for inaccuracies induced by unavoidable mechanical tolerances. Therefore, in case of optical systems, the authors adapted ideas of classical process planning in an intermediate step describing the desired geometric and especially the desired optical relations of the product parts for bridging the gap between product development and assembly execution. According to the terminology defined in [7] for mechanical assemblies, constraints or requirements on geometric and mechanical relations are named key characteristics (KC's).

KC's express the objectives and constraints of individual assembly steps as well as a quality measure for the whole assembly. This paper proposes to additionally include optical functions in the set of key characteristics. One important difference compared to plain mechanical assemblies is that constraints can be defined by parts that do not have a direct physical linkage. The modeling environment needs to support these circumstances. Furthermore, this paper shows how to formally define dependencies between KC's allow the derivation of the sequence of assembly steps (assembly sequence graph or precedence graph) and therefore the generation of platform independent assembly logic. KC's defining the desired relation between certain parts can be modeled hierarchically in order to derive mechanical constraints from optical ones. This information completes the assembly logic. 

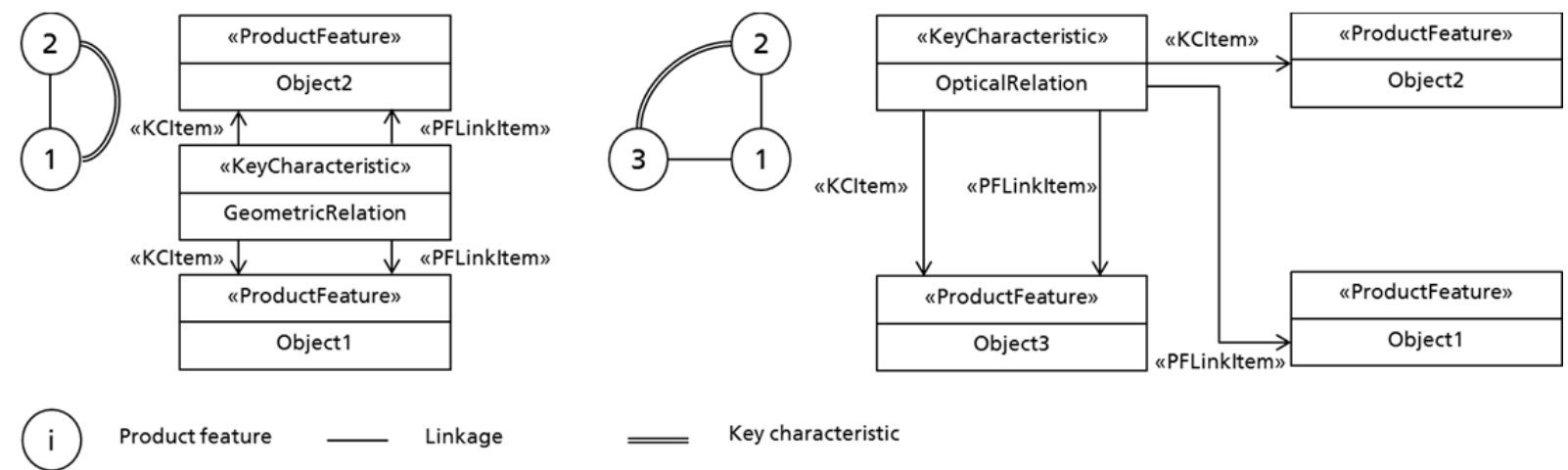

Product feature

Linkage

Key characteristic

Fig. 4: Formalized target configuration patterns as liaison diagram and as formalized liaison diagram: key characteristic directly specifying the constraints on a physical linkage between two product features (left), key characteristic specifying the constraints on a physical linkage between two product features using a third product feature (right).

Fig. 3Fehler! Verweisquelle konnte nicht gefunden werden. shows two patterns of describing physical linkages between product features such as gluing areas or optical surfaces. Two types of representations are used in the figure. One representation (liaison diagram) uses a number code representing a product feature. The solid line connecting two product features stands for a physical linkage, the double line stands for a key characteristic specifying the constraints of the physical linkage. A constraint could be a specific geometric relation or an optical function. This kind of representation is applicable for intuitively sketching out target configurations.

The second representation introduced by the authors is a formal representation of a liaison diagram (formalized liaison diagram) and it uses a newly defined UML profile and is semantically equivalent to the first one. Extending UML allows the use of standard computer-aided software engineering (CASE) tools. State-of-the-Art UML tools allow the application of text templates on such diagrams for generating machine readable code which can be processed further within the subsequent engineering tool-chain.

By definition in this paper, one $\mathrm{KC}$ physically links two product features (PFLinkItem). Furthermore, a KC defines a geometric or a functional relationship. In order to describe such a relationship two more product features are referenced as key characteristic items (KCItem). KCItems and PFLinkItems can be the same product features as depicted in Fig. 4Fehler! Verweisquelle konnte nicht gefunden werden., left. This is the case when, for example, an optical element is bonded onto a carrier plate using geometric features such as parallelism and distance of edges. Sometimes it is necessary to physically link two product features while the key characteristic such as an optical function is defined by other items. Fig. 4Fehler! Verweisquelle konnte nicht gefunden werden., right, shows a configuration where Object1 (carrier) holds Object2 (lens) and Object2 is attached to Object3 (e.g. heat sink of a laser diode bar). The constraint on the physical linkage between Object1 and Object 3 is determined by the optical function between Object3 and Object 2 .

The alignment of micro-optical components fulfilling optical constraints often requires multiple steps. Firstly, the positioning system places the optical component using machine accuracy (pre-positioning). Secondly, the component is aligned according to geometric features (passive alignment) using machine vision and e.g. edge detection. Thirdly, many optical components require active alignment for directly optimizing the desired optical function. Fig. 5 shows an optical KC which is refined by other key characteristics (KCRefinement).

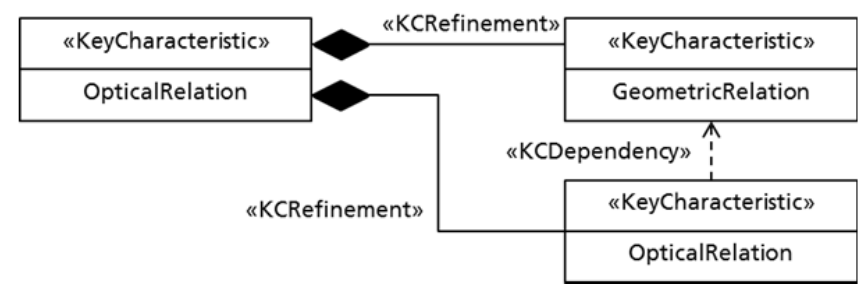

Fig. 5: Refinement of key characteristics (KCRefinement) and dependencies between key characteristics

Using the proposed UML profile, dependencies between key characteristics can be expressed. This is required for describing multi-step alignment (pre-positioning, passive alignment, active alignment). The combination of the features of refinement and dependency allows the expression of highly complex assembly constraints carrying all relevant information for subsequent assembly execution. Based on the formalized product configuration description the assembly sequence can be derived as explained in the following section.

\subsection{Derivation of the assembly sequence and commissioning}

The assembly sequence can be derived from the dependencies between key characteristics by evaluating the KCRefinement and KCDependency relation stereotypes in the formalized liaison diagram. The resulting 
assembly sequence describes the assembly task in a platform-independent way because the assembly platform is not part of this description (vPIPP) and therefore no platform specific information is available. Neither resources such as manipulators and grippers nor coordinates are specified yet. Active alignment routines and other complex process steps are represented by placeholders at first. Within the context of the work presented, different tools are under development for efficiently planning sensor-based processes in a hybrid assembly process planning system.

Once an alignment strategy is referenced as a subroutine by the placeholder its functionality is verified by an alignment-in-the-loop software tool. This tool uses the application programming interface of the commercial optical modelling and simulation software for manipulating the position and orientation of the lens under inspection. Output of the simulation is a simulated intensity profile of the optical setup which is processed by the alignment routine calculating corrective values for manipulating the lens. For the verification of alignment strategies, the lens under inspection is initialized randomly regarding its position and orientation and then the steps of processing the intensity profile, calculating the corrective value, and manipulating the lens virtually are carried out in a loop until the termination criteria is reached.

The transfer to a production platform requires two major steps: 1. teaching of coordinates in the VPS, 2 . assigning reference mark detection routines. Teaching and planning based on a VPS minimizes standstill times of the physical production system. Fig. 6 shows different shapes of reference marks which can be detected in a standard way. This allows a mapping between model data and real world data during process execution.
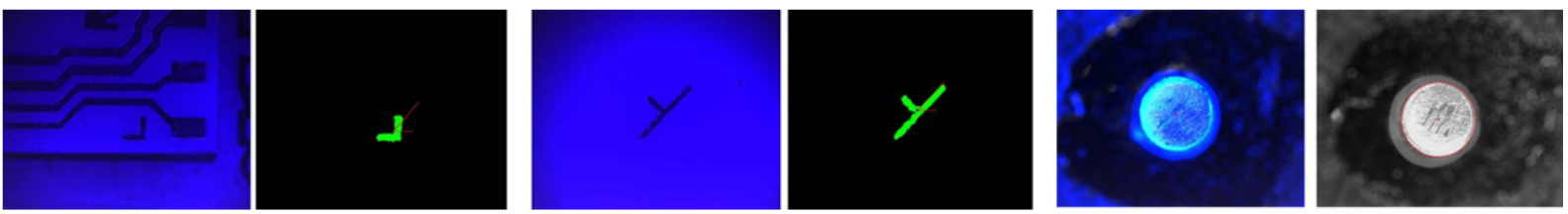

Fig. 6: Samples of reference marks with corresponding results from image-processing (from [4]).

\section{Exemplary assembly specification}

This section illustrates the application of the proposed formalism for specifying the assembly task of a collimation module for diode laser systems. The optical system consists of a fast-axis collimation lens (FAC) and a slow-axis collimation lens (SAC). The optics have to be aligned in a specific distance in order to fulfill the optical function of the module. Two side tabs are used for holding the optical components. The side tabs do not have an optical function. Fig. 7 shows the target configuration of the collimation module as a wire frame model (middle) and its representation in a liaison diagram (left).
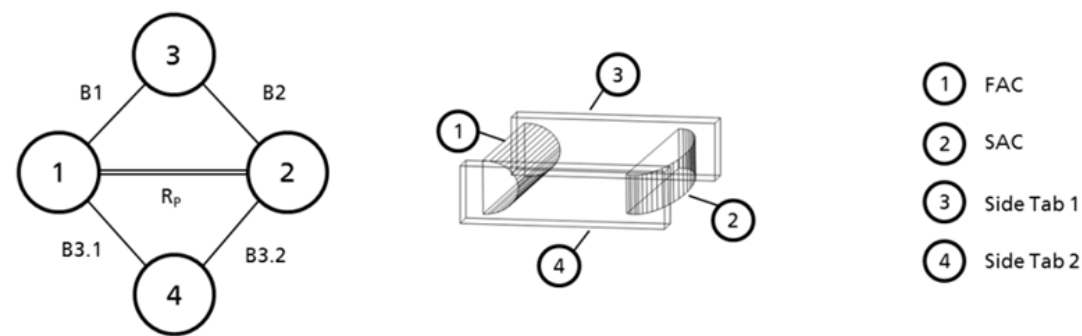

Collimation module

Fig. 7: Representation of the target configuration of a collimation module. The wire frame sketch (middle) illustrates the optical system. The left column shows the formal representation.

Fig. 8 depicts the assembly sequence of the collimation module. The additional nodes (A) and (B) represent mechanical end stops used for passive referencing. The dashed lines represent temporal linkages used during assembly. In step (a), the side tab is aligned temporarily using the mechanical endstops (A) and (B). In step (b), the FAC is inserted temporarily. In (c), the linkage is controlled by a key characteristic applying geometric constraint. In (d), the SAC is assembled regarding a geometric constraint between FAC and SAC. Steps (e) and (f) add the second side tab to the module. Picture (g) represents the final product with the fulfilled constraint. 


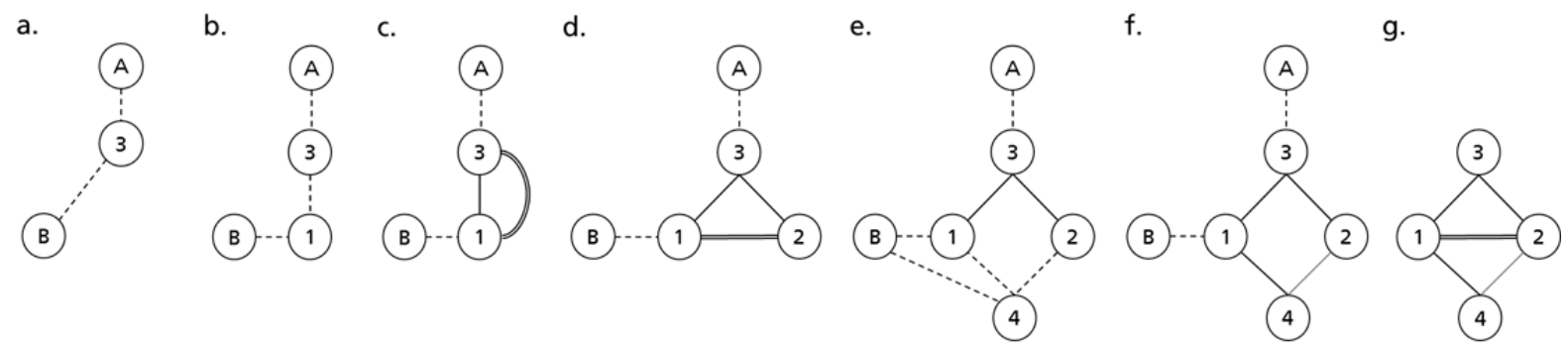

Fig. 8: Individual assembly steps of the collimation module with side tabs.

\section{Conclusion and outlook}

This paper introduced a formal notation for specifying target configurations of micro-optical systems. The notation includes information about key characteristics which determine the optical function of the system as well as the constraints of each assembly step. Key characteristics can be refined in order to match the multistep alignment approach for optical systems comprising the steps of pre-positioning, passive alignment, and active alignment. Furthermore, the definition of dependencies between key characteristics can be exploited in order to generate a platform-independent assembly sequence.

Future work will be dedicated to the realization of a tool-chain by implementing software tools for specifying the individual assembly steps in a level of detail which allows their execution through an assembly platform. The focus will be put on the specification of strategies for sensor-guided process steps and especially active alignment processes as the engineering of such processes is currently the bottleneck regarding the planning and commissioning of micro-optical assembly.

\section{Acknowledgement}

The authors would like to thank the German Federal Ministry of Education and Research (BMBF) for the support of the work by financing the MANUNET-DeLas project (funding code 02PJ2542).

\section{References}

[1] Brecher, Christian (Ed.) (2012): Integrative production technology for high-wage countries. Berlin, New York: Springer.

[2] Brecher, C.; Pyschny, N.; Haag, S.; Guerrero Lule, V. (2012): Automated alignment of optical components for high-power diode lasers. In: Mark S. Zediker (Hg.): High-power diode laser technology and applications X. 22-24 January 2012, San Francisco, California, United States. Bellingham, Wash: SPIE (Proceedings of SPIE, v. 8241), S. 82410D-82410D-11.

[3] Brecher, C.; Pyschny, N.; Haag, S.; Mueller, T. (2013): Automated assembly of VECSEL components. In: Jennifer E. Hastie (Hg.): Vertical external cavity surface emitting lasers (VECSELs) III. 3-5 February 2013, San Francisco, California, United States (Proceedings of SPIE, volume 8606), S. 86060I-86060I14.

[4] Garlich, Torsten; Guerrero, Vicente; Hoppen, Martin; Müller, Tobias; Pont, Pere; Pyschny, Nicolas et al. (2012): SCALAB. Scalable Automation for Emerging Lab Production. Final report of the MNT-ERA.net research project. Apprimus-Verl.

[5] Miesner, Jörn; Timmermann, Andre; Meinschien, Jens; Neumann, Bernhard; Wright, Steve; Tekin, Tolga et al. (2009). In: Mark S. Zediker (Hg.): High-power diode laser technology and applications VII. 26-27 January 2009, San Jose, California, United States. Bellingham, Wash: SPIE (v. 7198), S. 71980G71980G-11.

[6] Pierer, J.; Lützelschwab, M.; Grossmann, S.; Spinola Durante, G.; Bosshard, Ch.; Valk, B. et al. (2011). In: Mark S. Zediker (Hg.): High-power diode laser technology and applications IX. 23-25 January 2011, San Francisco, California, United States. Bellingham, Wash: SPIE (v. 7918), S. 79180I-79180I-8.

[7] Whitney, Daniel E. (2004): Mechanical assemblies. Their design, manufacture, and role in product development. New York: Oxford University Press.

[8] Scheller, Torsten (2001): Analysis of Automated Assembly Processes for Hybrid Micro-optical Systems (German original: Untersuchung zu automatisierten Montageprozessen hybrider mikrooptischer Systeme). Dissertation.

[9] Müller, Rainer; Riedel, Martin; Vette, Matthias; Corves, Burkhard; Esser, Martin; Hüsing, Mathias (2010): Reconfigurable Self-optimising Handling System. In: Svetan Ratchev (Ed.): Precision Assembly Technologies and Systems, pp. $255-262$.

[10] Gottipolu, R. B.; Gosh, K. (1997): Representation and Selection of Assembly Sequences in Computeraided Assembly Process Planning. In: International Journal of Production Research 35 (12), pp. 3447- 
Integrated Tool-chain Concept

for Automated Micro-optics Assembly

3466.

[11] ElMaraghy, Hoda A. (Ed.) (2009): Changeable and Reconfigurable Manufacturing Systems. London: Springer London (Springer series in advanced manufacturing).

[12] Gašević, Dragan; Djurić, Dragan; Devedzic, Vladan (2009): Model driven engineering and ontology development. 2nd ed. Dordrecht, New York: Springer. 